\title{
A Literature Review of Emotional Labor and Non-Task Behavior
}

\author{
Yeong-Gyeong Choi", Kyoung-Seok Kim
}

Business Administration, Kyungpook National University, South Korea

Copyright $@ 2015$ Horizon Research Publishing All rights reserved.

\begin{abstract}
This study, literature review research, intends to deal with the problem of conceptual ambiguity among research on emotional labor, and to look into the evolutionary trends and changing aspects of defining the concept of emotional labor. In addition, in existing studies, deep acting and surface acting are highly related to a positive outcome variable and a negative outcome variable, respectively. It was confirmed that for employees performing emotional labor, deep acting and surface acting are highly related to $\mathrm{OCB}$ and $\mathrm{CWB}$, respectively. While positive emotion that employees come to experience during job performance process can easily trigger a positive non-task behavior such as OCB, negative emotion that employees experience through excessive workload or unfair treatment can easily induce a negative behavior like CWB. The two management behaviors of emotional labor, surface acting and deep acting, can have either a positive or negative effect on non-task behavior of employees, depending on which one they would choose. Thus, the purpose of this review paper is to clarify the relationship between emotional labor and non-task behavior more specifically.
\end{abstract}

Keywords Emotion Labor, Non-Task Behavior, OCB, CWB

\section{Introduction}

Due to the rapid change of organizational environments, there is a growing concern that if employees perform only official works, it could lead to deterioration of organizational dynamism and competitiveness, and the importance of non-task behavior, that is, discretionary behavior of employees, is being emphasized. Studies on non-task behavior can be largely divided into OCB (organizational citizenship behavior) and CWB (counterproductive work behavior).

OCB is drawing much attention as a variable that improves organizational effectiveness. Although OCB is not directly acknowledged by the official compensation system of an organization, it is defined as a voluntary behavior of an individual contributing to the effective management of an organization, and as a behavior that helps organizational effectiveness by supporting smooth job performance even though it is not officially specified in the job description. If OCB is a positive non-task behavior, CWB is a typical negative non-task behavior. CWB not only influences job performance of individuals negatively, but is an intentional and voluntary behavior that aggravates effectiveness of the whole organization, and thus, it is interpreted in the similar context to misdeed within an organization, deviant behavior, or dysfunctional behavior.

Managing non-task behavior of employees in the organizational level is an important task to improve organizational performance, and for employees, OCB should be appropriately encouraged and CWB should be actively prevented and reduced.

As presently the importance of the emotion that organizational members experience, as one of the factors of non-task behavior occurrence, is being focused, empirical researches to specify the relationship between emotional labor and non-task behavior are increasing.

In other words, while positive emotion that employees come to experience during job performance process can easily trigger a positive non-task behavior such as OCB, negative emotion that employees experience through excessive workload or unfair treatment can easily induce a negative behavior like CWB.

The two management behaviors of emotional labor suggested by Hochschild(1983), surface acting and deep acting, can have either a positive or a negative effect on non-task behavior of employees, depending on which one they would choose.

That is, although the two variables have a common ground that they both pursue emotional labor in the same direction as organizational normative emotion, their intentions and attributes for emotional labor are distinctively different, and actually these two concepts have very different influences on non-task behavior.

In the emotional labor field, there are mixed studies reporting that emotional labor could have a positive or a negative effect on task behavior and task performance of employees. This is because they are explaining the positive 
and negative effects of emotional labor from their own viewpoints, and their logical developments are not clear.

Thus, the purpose of this review paper is to clarify the relationship between emotional labor and non-task behavior more specifically.

\section{Main body}

\subsection{Emotional Labor}

A scholar who first had interest in emotional labor was Hochschild(1979, 1983). Before Hochschild, 'emotion' was recognized as a simple instinctive aspect of human beings or a sociocultural construct. However, he defined emotion as a labor unit that can be exchanged with goods, and designated a behavior to manage and do desirable emotional expression required by an organization as emotional labor[1].

Hochschild, based on Goffman's study (1959), explained emotional labor in the dramaturgical perspective related to a social interaction. Performers express their intention properly, represent themselves effectively, and manage their impression efficiently to show good impression to audiences. Thereby, He represented customers as audiences, employees as performers, and job environments as stages, and claimed that employees try to satisfy audiences by controlling their emotion.

He used the new type of labor, emotional labor, not physical labor or mental labor. In order to understand the concept of emotional labor, a few aspects shall be considered.

\subsection{Definition of Emotional Labor}

Since the concept of emotional labor introduced by Hochschild(1983), efforts to refine the concept of emotional labor have been made by many researchers(Ashforth \& Humphrey, 1993; Grandey, 2000; Morris \& Feldman, 1996)[2].

Hochschild defined emotional labor as "the management of feeling to create a publicly observable facial and bodily display; emotional labor is sold for a wage and therefore has exchange value"(Hochschild, 1983)[1]. According to Hochschild, jobs with emotional labor have three criteria;1)they require face-to-face or voice-to-voice contact with the public 2)they require the worker to produce an emotional state in the client or customer 3)they allow the employer, through training and supervision, to exercise a degree of control over the emotional activities of employees(Hochschild, 1983).

Ashforth \& Humphrey(1993) contributions to the area of emotional labor have propelled the study of emotions forward, since they include the role of social identity and integrate it into the study of emotions and their expression at work. They defined emotional labor as "the act of displaying the appropriate emotion"(Ashforth \& Humphrey, 1993).
Morris \& Feldman(1996, 1997) have also contributed to the growing literature on emotional labor in organizations by refining the conceptualization of emotional labor. They defined emotional labor as "the effort, planning, and control needed to express organizationally desired emotion during interpersonal transaction"(Morris \& Feldman, 1996).

Grandey(2000) provides another conceptualization of emotional labor in an attempt to clear up the apparent contradictions resulting from attempts in the literature to refine the construct of emotional labor. Grandey(2000) defined emotional labor as "the process of regulating both feelings and expressions for organizational goals".

Table 1. Definition of Emotional Labor

\begin{tabular}{|c|l|}
\hline Scholar & \multicolumn{1}{|c|}{ Definition } \\
\hline $\begin{array}{c}\text { Hochschild } \\
(1983,1989)\end{array}$ & $\begin{array}{l}\text { the management of feeling to create a publicly } \\
\text { observable facial and bodily display }\end{array}$ \\
\hline $\begin{array}{c}\text { Ashforth \& } \\
\text { Humphrey } \\
(1993)\end{array}$ & the act of displaying the appropriate emotion \\
\hline $\begin{array}{c}\text { Morris \& } \\
\text { Feldman } \\
(1996,1997)\end{array}$ & $\begin{array}{l}\text { the effort, planning, and control needed to } \\
\text { express organizationally desired emotion } \\
\text { during interpersonal transaction }\end{array}$ \\
\hline $\begin{array}{c}\text { Grandey } \\
(2000)\end{array}$ & $\begin{array}{l}\text { the process of regulating both feelings and } \\
\text { expressions for organizational goals }\end{array}$ \\
Krual \& Geddes & $\begin{array}{l}\text { what employees perform when they are } \\
\text { required to feel or at least project the } \\
\text { appearance of certain emotions in order to } \\
\text { produce }\end{array}$ \\
\hline $\begin{array}{c}\text { Diefendorff \& } \\
\text { Richard(2003) }\end{array}$ & $\begin{array}{l}\text { the management of emotions as part of the } \\
\text { work role }\end{array}$ \\
\hline Johnson & $\begin{array}{l}\text { the expression of organizationally desired } \\
\text { emotions by service agents during service } \\
\text { encounters }\end{array}$ \\
\hline
\end{tabular}

Looking at it in the static/dynamic aspects, its expressional principle is static. Emotional expression during individual works should meet the social norm first. Each industry has its own unique industrial guideline. Thus, emotional expression during individual works should satisfy this industrial guideline. Although these social norm and industrial guideline are static, recognition on, comparison of, and compliance with the expressional principle require dynamic emotion management and emotion control of employees.

In the organizational aspect, an organization requires its members to express their emotion appropriately. Therefore, what an organization gives weight to is external emotional expression. It does not consider their internal emotion important. In the individual aspect, employees try to change and control their emotion for their economic advantages.

In the external aspect, emotional labor is mainly expressing external emotion. In the internal aspect, emotional labor is a series of complex and dynamic psychological regulative process.

In the emotional environmental aspect, emotional labor occurs from the job performing condition, and is included in personal relations. Emotional labor is, among employees' interactions with customers, individual emotional expression influencing others' emotion, which enables proper completion of job performance. 


\subsection{Surface Acting \& Deep Acting}

The two strategies suggested by Hochschild(1983), deep acting and surface acting, have a common ground in that they both are a kind of complementary emotional labor strategy that employees use when they cannot naturally express their emotion (Diefendorff, Croyle\&Gosserand, 2004), and both the two let employees perform emotional labor in the same way as normative emotion that an organization requires them to express to customers (Ashforth\& Humphrey, 1993; Grandey, 2003).

However, intentions of the two strategies are definitely different from each other.

Surface acting is to make only the visual aspect of employees' emotion correspond to the emotional expression principle of an organization. Accordingly, emotional dissonance could be easily induced (Ashforth\& Humphrey, 1993; Brotheridge\&Grandey, 2002; Grandey, 2003; Zapf, 2002)[3].

Deep acting is not only to create externally expressed emotion but to change internal emotion in the direction of the emotional expression principle. For this, empathy, and thoughts and images that trigger specific emotion are actively utilized (Ashforth\& Humphrey, 1993; Brotheridge\&Grandey, 2002; Grandey, 2003; Zapf, 2002).

The two emotional labor strategies suggested by Hochschild(1983), deep acting and surface acting, have a common ground that both the two attempt to perform emotional labor in the same way as normative emotion that an organization requires employees to express, when they cannot appropriately express their emotion (Ashforth\& Humphrey, 1993; Grandey, 2003).

However, these two emotional labor strategies have a clear difference. The surface acting strategy attempts to make only external expressions correspond to normative emotion required by an organization regardless of employees' internal emotion. This could cause emotional dissonance due to conflicts between employees' internal feeling and normative emotion(Rafaeli\& Sutton, 1987). In this light, the surface acting strategy is different from the deep acting strategy, and can be named 'fake in bad faith'. The deep acting strategy tries to change even employee's internal emotion in the direction of normative emotion. In this regard, the deep acting strategy can be named 'fake in good faith'(Rafaeli\& Sutton, 1987).

Existing studies showed that surface acting results in negative emotional labor and deep acting results in positive emotional labor. For example, they reported that while surface acting is related to negative results such as depersonalization, emotional exhaustion, or dissatisfaction, deep acting is related to positive results such as sincerity or a sense of fulfillment (Brotheridge\&Grandey, 2002; Brotheridge\& Lee, 2002, 2003; Diefendorff\&Gosserand, 2003; Grandey, 2003; Grandey et al, 2005)[4].

Deep acting and surface acting have different results and effectiveness. That is, because while deep acting is not only to create externally expressed emotion but to change even internal emotion in the direction of the emotional expression principle, surface acting is only to make the visual emotional aspect correspond to the organizational emotional expression principle (Hochschild, 1983), deep acting is highly relevant to a positive outcome variable and surface acting is highly relevant to a negative outcome variable.

Grandey(2000) suggested a conceptual model by using emotion regulation theory through characterizing emotional labor. This emotion regulation theory was suggested by Gross(1998), which indicates 'the process that influences what emotion individuals have, when they feel that emotion, how they experience emotion, and how they express it'. Grandey(2000) emphasized the importance of surface acting and deep acting during the process of performing emotional labor. That is, he argued that surface acting and deep acting could bring about both positive and negative results upon emotional labor[6]. For example, surface acting could have a negative effect on job satisfaction due to the discord that individuals experience. On the other hand, deep acting could positively influence job satisfaction because it makes individuals feel a sense of fulfillment that they have expressed their emotion appropriately. Grandey(2000) includes not only the outcome variable of emotional labor but situational, individual, and organizational factors that influence emotional labor.

\subsection{Dimensions of Emotional Labor}

Hochschild discussed surface acting and deep acting as key features of emotional labor, however, several researchers suggested that emotional labor is a multidimensional construct and may involve strategies other than surface acting and deep acting.

Ashforth \& Humphrey(1993) argued that employees must do surface acting or deep acting in order to express the expected emotions. However, they took the definition of emotional labor a step further by including a third category of emotional labor called expression of genuine emotion. They stated that conceptualizing emotional labor as surface acting and deep acting alone dismisses the possibility of employees spontaneously and genuinely experiencing and displaying appropriate emotions.

Morris \& Feldman(1996) conceptualize emotional labor in terms of four distinct dimensions; 1)the frequency of appropriate display 2)attentiveness to required display rules 3)variety of emotions required to be displayed 4)the emotional dissonance generated as a result of having to express organizationally desired emotions that are not genuinely felt. Later, in their empirical research on antecedents and consequences of emotional labor, emotional labor was identified into three dimensions: 1)frequency 2)duration 3)emotional dissonance.

Brotheridge \& Lee(2003) developed the six faceted Emotional Labor Scale(ELS) based on the theories of Hochschild(1983) and Morris \& Feldman(1996). Their ELS measures the "intensity, frequency, variety of emotional display, surface acting, deep acting and the duration of 
interaction.

\subsection{Consequences of Emotional Labor}

Starting with Hochschild(1979, 1983), negative and positive consequences of emotional labor for individuals and organizations have been suggested in the literature. Ashforth \&Humphrey(1993) described emotional labor as a double-edged sword.

Negative Consequences: In terms of the consequences of emotional labor, prior research mainly focuses on the potentially psychologically damaging effects on the employees who perform emotional labor. The most-often-cited consequences are emotional dissonance and job dissatisfaction.

Researchers express that, surface acting is likely to lead to emotional dissonance(Hochschild, 2003), and emotional exhaustion(Grandey, 2003). Surface acting is linked to burnout and lower service performance(Brotheridge \& Grandey, 2002). Heuven \& Bakker(2003) emphasized the importance of emotion work variables on emotional dissonance.

Parkinson(1991) argued that when employees' genuine feelings are masked, it leads to increase in job dissatisfaction. Abraham(1998) argued that increase in emotional dissonance increases job dissatisfaction.

Morris \& Feldman(1997) found that greater emotional dissonance, which is a form of estrangement of self and work role, is significantly associated with increased emotional exhaustion and job dissatisfaction.

Positive Consequences: Ashforth \& Humphrey(1993) proposed that expression of positive emotions is related to increased task effectiveness. Pugh(2001) remarked that the display of positive emotions by the employee is positively related to the customers' positive affect and this leads to positive evaluations of service quality[5].

Most of studies have yielded inconsistent results. It seems that the confusion with regarding to consequences of emotional labor stems from the fact that different definitions of the construct have been utilized with different antecedents by previous researchers.

Another reason for the contradictory conclusions about emotional labor's consequences is that researchers have failed to take into account the importance of individual factors. Researchers have posited that individual characteristics may play a primary role in explaining variation in the consequences(Rafaeli \& Sutton, 1989; Morris \& Feldman, 1997; Jones, 1998; Wharton, 1999)[7]. Therefore, taking individual characteristics into account as the antecedents of emotional labor can help understand how individuals perform emotional labor and its associated consequences.

\section{Emotional Labor and Non-Task Behavior}

The influence of emotional labor that has been mainly researched in relation to the official task behavior of employees engaged in the service industry could be expanded in the aspect of non-task behavior of organizational members. First, recent researches are newly emphasizing the importance of emotion that organizational members experience, as one of the factors that mediate the occurrence of non-task behavior. In other words, while positive emotion that organizational members come to experience during the job performance process can easily trigger positive non-task behavior such as $\mathrm{OCB}$, negative emotion that they experience due to heavy workload or unfair work condition can easily induce negative behavior such as CWB.

The possibility of occurrence of non-task behavior that is not officially required in an organization such as OCB or CWB could be expected in the social exchange relationship between the organization and its members. That is, according to social exchange theory, organizational members would have expectation and psychological contract notion from their organization, and as they experience emotional labor, they could easily feel unfairness that their emotional balance gets broken. In this case, it is likely that organizational members would preferentially select and use their discretionary behaviors rather than official job behaviors to recover their emotional balance. That is, it is highly possible that they would reduce their altruistic behaviors or voluntary contribution, or seek their psychological and economic compensation through CWB. In short, their discretionary behavior becomes the strategic means for recovering their well-balanced social exchange relationship with their organization

\subsection{Correlation between Emotional Labor and OCB}

There are few studies on the correlation between emotional labor and $\mathrm{OCB}($ Salami, 2009). As the difference between employees' actual emotion and that required by expressional norm increases, more effort and energy come to be needed, which leads to more stress and emotional dissonance. Because OCB starts from the theory that job satisfaction leads to organizational satisfaction resulting in contribution to the organization (Organ \& Ryan, 1995),surface acting could negatively influence OCB[8]. However, deep acting that accompanies active effort to change emotion could have a low level of emotional dissonance. Deep acting is to make an effort to change emotion by actively accepting organizational requirements, and this effort can be understood as devotion to the organization.

Existing studies related to the correlation between emotional labor and OCB are as below.

Salami(2007), by using the measurement tool of Podsakoff \& Mackenzie(1994) and three items, helping behavior, sportsmanship, and civic virtue, suggested that surface acting is negatively (-) correlated with OCB and deep acting is positively $(+)$ correlated with $\mathrm{OCB}$. And he also 
pointed out the need to study the influence of emotional labor on OCB, and claimed that OCB improves the effective function of an organization and is related to organizational effectiveness and success.

This is because most service-oriented organizations depend on direct face-to-face interactions between customers and employees (Abraham, 1998; Podsakoff et al., 1997). That is, emotional labor has been known to contribute to the general customer recognition on an organization and its products (Abraham, 1998; Diefendorff\& Richard, 2003)[9].

\subsection{Correlation between Emotional Labor and CWB}

Non-task behavior that influences organizational performance includes negative behaviors as well as positive behaviors such as OCB. Studies on negative non-task behavior have focused on deviant behavior, misdeed within an organization, CWB, etc. Although their researchers' theoretical viewpoints and backgrounds are different, their definitions and contents are generally similar, and presently those on CWB are being actively conducted.

During the process that employees seek their emotional balance in an organization, not performing $\mathrm{OCB}$ is not directly related to performing $\mathrm{CWB}$, and in turn, not performing CWB is not directly related to performing OCB. Because employees interacting with their organization independently select positive non-task behavior or negative non-task behavior, during the job performance process, positive emotion could make employees select positive non-task behavior such as OCB, and negative emotion could make them select negative non-task behavior such as CWB (Miles et al, 2002: Spector \& Fox, 2002).

Therefore, because if employees performing emotional labor understand customers, service recipients, and perform deep acting to help them during the job performance process, they come to try to make an effort for customers, their organization, and their own performance and career management, they will pursue $\mathrm{OCB}$ and reject $\mathrm{CWB}$ in performing non-task behavior.

\section{Implications}

Looking at most of the studies related to emotional labor, Hochschild's(1983) classification of deep acting and surface acting is applied because emotional labor itself focuses on the regulation of emotion.

Examining existing papers, it could be found that they have differences in results and effectiveness according to the type of deep acting and surface acting. The reason existing studies have different emotional labor results is the internal contradiction of the sub-levels of emotional labor. It is needed to conduct verification by dividing the sub-levels of emotional labor in detail.

In addition, in existing studies, deep acting and surface acting are highly related to a positive outcome variable and a negative outcome variable, respectively. It was confirmed that for employees performing emotional labor, deep acting and surface acting are highly related to OCB and CWB, respectively. Thus, human resource management that meets the characteristic of emotional labor should be realized.

Based on the results of existing papers that OCB could be raised through deep acting, an effort to improve utilization of human resources and organizational effectiveness by managing employees' emotional labor would be necessary. Of course, the best way would be reducing emotional labor through organizational supports or helps of colleagues or seniors. It is needed to encourage employees to seek deep acting than surface acting that brings about negative results such as emotional dissonance due to stress.

\section{REFERENCES}

[1] A. R.Hochschild, The Managed Heart, Berkeley \& Los Angeles, CA: University of CaliforniaPress, 1983.

[2] Ashforth, B. E., \& Humphrey, R. H(1993). Emotional labor in service roles: The influence of identity. Academy of Management Review, 18(1), 88-115.

[3] Brotheridge, C. M., \& Grandey, A. A(2002). Emotional labor and burnout: Comparing two perspectives of "people work". Journal of Vocational Behavior, 60, 17-39.

[4] Diefendorff, J. M., \& Gosserand, R. H(2003). Understanding the emotional labor process: A control theory perspective. Journal of Organizational Behavior, 24, 945-959.

[5] Fredrickson, B. L(2001). The role of positive emotions in positive psychology: The broaden-and-build theory of positive emotions. American Psychologist, 56 (3), 218- 226.

[6] Grandey, A. A(2000). Emotion regulation in the workplace: A new way to conceptualize emotional labor. Journal of Occupational and Health Psychology, 5(1), 95-110.

[7] Morris, J. A., \& Feldman, D. C(1996). The dimensions, antecedents, and consequences of emotional labor. Academy of Management Review, 21(4), 986-1010.

[8] Daily, B.F., Bishop, J.W. \& Govindarajulu, N. (2009) A conceptual model for organizational citizenship behavior directed toward the environment, Business \& Society, 48(2), 243-256.

[9] Lavelle, J., Rupp, D., \& Brockner, J. (2007) Taking a multifoci approach to the study of justice, commitment, and organizational citizenship behavior: The target similarity model, Journal of Management, 33(6), 841-866. 